\title{
Algoritmo para o Fator de Esquecimento do Método Cepstral de Cancelamento de Realimentação Acústica
}

\author{
Wellington M. da S. Nogueira, Cézar F. Yamamura, Bruno C. Bispo, Edson A. R. Theodoro, Pedro M. Rodrigues
}

Resumo-Este trabalho propõe uma melhoria no método de cancelamento de realimentação acústica baseado no cepstro do sinal de erro. Essa melhoria consiste em um algoritmo para transformar o fator de esquecimento, parâmetro que controla o compromisso entre robustez a perturbações de curta duração e capacidade de rastreamento do filtro adaptativo, em variante no tempo. Simulações demonstraram que esse algoritmo faz o método apresentar um melhor compromisso entre velocidade de (re-)convergência e limite de desalinhamento, aumentando de modo geral a margem de estabilidade do sistema de sonorização.

Palavras-Chave-Cancelamento de realimentação acústica, método cepstral, fator de esquecimento.

Abstract-This work proposes an improvement in the acoustic feedback cancellation method based on the cepstrum of the error signal. This improvement consists of an algorithm to transform the forgetting factor, parameter that controls the tradeoff between robustness to short-burst disturbances and tracking rate of the adaptive filter, into time-varying. Simulations showed that this algorithm makes the method present a better trade-off between (re-)convergence speed and misalignment limit, generally increasing the stability margin of the sound reinforcement system.

Keywords-Acoustic feedback cancellation, cepstrum-based method, forgetting factor.

Notação: Os símbolos $n$ e $q$ denotam o índice de tempo discreto e o operador de deslocamento, respectivamente, tal que $q^{-1} x(n)=x(n-1)$ [1]. Um filtro de tempo discreto e com comprimento $L_{F}$ é representado pela função de transferência variante no tempo [1]

$$
F(q, n)=f_{0}(n)+f_{1}(n) q^{-1}+\ldots+f_{L_{F}-1}(n) q^{-L_{F}+1}
$$

ou pela sua resposta ao impulso

$$
\mathbf{f}(n)=\left[\begin{array}{llll}
f_{0}(n) & f_{1}(n) & \ldots & f_{L_{F}-1}(n)
\end{array}\right] .
$$

A filtragem do sinal $x(n)$ com $F(q, n)$ é denotada como

$$
F(q, n) x(n)=\mathbf{f}(n) * x(n)=\sum_{k=0}^{L_{F}-1} f_{k}(n) x(n-k) .
$$

A transformada de Fourier de tempo curto de $\mathbf{f}(n)$ é indicada por $F\left(e^{j \omega}, n\right)$ onde $\omega \in[0,2 \pi]$ é a frequência angular.

\section{INTRODUÇÃO}

Um sistema de sonorização típico utiliza microfone(s), sistema de amplificação e alto-falante(s) para captar, amplificar e reproduzir, respectivamente, o sinal da fonte $v(n)$ no mesmo ambiente acústico. Desconsiderando o filtro adaptativo, esse

Wellington M. da S. Nogueira, Cézar F. Yamamura, Bruno C. Bispo e Edson. A. R. Theodoro, Departamento de Engenharia Elétrica, Universidade Tecnológica Federal do Paraná, Cornélio Procópio-PR, Brasil. Pedro M. Rodrigues, Escola Superior de Biotecnologia, Universidade Católica Portuguesa, Porto, Portugal. E-mail: brunobispo@utfpr.edu.br.

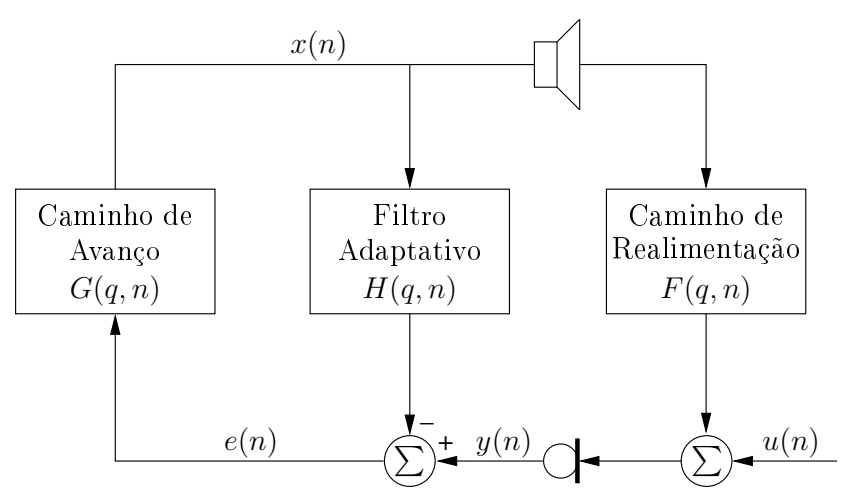

Fig. 1: Cancelamento de realimentação acústica.

sistema é ilustrado na Figura 1 [2], [3]. O acoplamento acústico entre alto-falante e microfone faz o sinal do altofalante $x(n)$ retornar ao microfone, gerando assim um laço fechado de sinal.

O caminho de realimentação modela o acoplamento acústico entre alto-falante e microfone e, por simplicidade, também inclui as características do conversor D/A, alto-falante, microfone e conversor $\mathrm{A} / \mathrm{D}$, sendo definido como um filtro $F(q, n)$. $\mathrm{O}$ caminho de avanço modela o sistema de amplificação e qualquer dispositivo de processamento de sinal inserido nesta parte do sistema, sendo definido como um filtro $G(q, n)$.

O sinal de entrada do sistema $u(n)$ é a soma do sinal da fonte $v(n)$ e do sinal de ruído ambiente $r(n)$, isto é, $u(n)=$ $v(n)+r(n)$. O sinal do alto-falante $x(n)$ está relacionado com o sinal de entrada do sistema $u(n)$ pela função de transferência em malha fechada do sistema da seguinte forma

$$
x(n)=\frac{G(q, n)}{1-G(q, n) F(q, n)} u(n) .
$$

O critério de estabilidade de Nyquist afirma que o sistema em malha fechada pode se tornar instável se houver pelo menos uma frequência $\omega$ para a qual

$$
\left\{\begin{array}{l}
\left|G\left(e^{j \omega}, n\right) F\left(e^{j \omega}, n\right)\right| \geq 1 \\
\angle G\left(e^{j \omega}, n\right) F\left(e^{j \omega}, n\right)=2 k \pi, k \in \mathbb{Z} .
\end{array}\right.
$$

Neste caso, o resultado é um som tipo apito, um fenômeno conhecido como efeito Larsen [2], [3]. Esse som tipo apito será muito incômodo para os ouvintes e o ganho de amplificação terá que ser reduzido. Como consequência, o máximo ganho estável (MSG) do sistema de sonorização tem um limite superior devido à realimentação acústica [2], [3].

Com o objetivo de quantificar a amplificação alcançável em um sistema de sonorização, costuma-se definir um ganho de 
banda larga do caminho de avanço como [2], [3]

$$
K(n)=\frac{1}{2 \pi} \int_{0}^{2 \pi}\left|G\left(e^{j \omega}, n\right)\right| \mathrm{d} \omega
$$

e extraí-lo de $G(q, n)$ da seguinte forma

$$
G(q, n)=K(n) J(q, n) .
$$

Assumindo que $J(q, n)$ é conhecido e $K(n)$ pode ser variado, o MSG do sistema de sonorização é definido como

$$
\begin{gathered}
\operatorname{MSG}(n)(\mathrm{dB})=20 \log _{10} K(n) \\
\text { tal que } \max _{\omega \in P(n)}\left|G\left(e^{j \omega}, n\right) F\left(e^{j \omega}, n\right)\right|=1,
\end{gathered}
$$

resultando em

$\operatorname{MSG}(n)(\mathrm{dB})=-20 \log _{10}\left[\max _{\omega \in P(n)}\left|J\left(e^{j \omega}, n\right) F\left(e^{j \omega}, n\right)\right|\right]$,

onde $P(n)$ denota o conjunto de frequências que satisfazem a condição de fase em (5), também chamadas de frequências críticas do sistema de sonorização, isto é,

$$
P(n)=\left\{\omega \mid \angle G\left(e^{j \omega}, n\right) F\left(e^{j \omega}, n\right)=2 k \pi, k \in \mathbb{Z}\right\} .
$$

Para controlar o efeito Larsen e aumentar o MSG, os métodos de cancelamento de realimentação acústica (AFC) identificam $F(q, n)$ utilizando um filtro adaptativo $H(q, n)$. Então, o sinal de realimentação $\mathbf{f}(n) * x(n)$ é estimado como $\mathbf{h}(n) * x(n)$ e subtraído do sinal do microfone $y(n)$ de forma que, idealmente, $e(n)=u(n)$ [2], [3]. Esse esquema é representado na Figura 1 e similar ao cancelamento de eco acústico (AEC) utilizado em sistemas de teleconferência [4].

Porém, no AFC, os algoritmos de filtragem adaptativa tradicionais, baseados no gradiente ou mínimos quadrados, apresentam desempenho insatisfatório porque os sinais que agem como entrada, $x(n)$, e interferência, $u(n)$, para o filtro adaptativo $H(q, n)$ são correlacionados [2], [3], [5], [6], [7].

$\mathrm{Na}$ sua maioria, as soluções existentes na literatura para superar esse problema tentam descorrelacionar o sinal do alto-falante $x(n)$ e o sinal de entrada $u(n)$ mas continuam utilizando os tradicionais algoritmos de filtragem adaptativa para atualizar $H(q, n)$ [2], [3].

Por outro lado, os recentes métodos AFC-CM e AFC-CE exploram definições do cepstro do sinal do microfone $y(n) \mathrm{e}$ do sinal de erro $e(n)$, respectivamente, em função de $\mathbf{g}(n)$ e $\mathbf{f}(n)$ a fim de calcular estimativas da resposta ao impulso do caminho de realimentação e utilizá-las para atualizar o filtro adaptativo $H(q, n)$ [3], [8], [9]. Esses métodos, em particular o AFC-CE, apresentam resultados muito promissores em diferentes configurações de sistemas de sonorização, incluindo aparelhos auditivos [3], [8], [10], [11], [12].

Esse trabalho propõe uma melhoria no método AFC-CE e a avalia utilizando sinais de voz. Este trabalho está organizado da seguinte maneira: a Seção II descreve resumidamente o método AFC-CE; na Seção III, uma melhoria no método é proposta; a Seção IV descreve a configuração do ambiente simulado; na Seção V, os resultados são apresentados e discutidos; por fim, a Seção VI conclui o trabalho.

\section{O MÉTODo AFC-CE}

No sistema representado na Figura 1 , se $\mid G\left(e^{j \omega}, n\right)$ $\left[F\left(e^{j \omega}, n\right)-H\left(e^{j \omega}, n\right)\right] \mid<1$, condição suficiente para garantir a estabilidade do sistema, então o cepstro de tempo curto do sinal de erro $e(n)$ pode ser definido como [3]

$$
\mathbf{c}_{\mathbf{e}}(n)=\mathbf{c}_{\mathbf{u}}(n)+\sum_{k=1}^{\infty} \frac{\{\mathbf{g}(n) *[\mathbf{f}(n)-\mathbf{h}(n)]\}^{* k}}{k},
$$

onde $\{\cdot\}^{* k}$ denota a $k$-ésima potência de convolução.

A partir de (11), conclui-se que o cepstro de tempo curto $\mathbf{c}_{\mathbf{e}}(n)$ do sinal de erro é o cepstro de tempo curto $\mathbf{c}_{\mathbf{u}}(n)$ do sinal de entrada do sistema adicionado à uma série em função de $\mathbf{g}(n), \mathbf{f}(n)$ e $\mathbf{h}(n)$. Essa série é formada por convoluções sucessivas de $\mathbf{g}(n) *[\mathbf{f}(n)-\mathbf{h}(n)]$, a resposta ao impulso em malha fechada do sistema de sonorização.

O método AFC baseado no cepstro do sinal do erro (AFCCE) calcula $\mathbf{c}_{\mathbf{e}}(n)$ utilizando as $L_{f r}$ amostras mais recentes de $e(n)$ [3]. Então, o método calcula $\{\mathbf{g} *[\mathbf{f}(n)-\mathbf{h}(n)]\}$, uma estimativa de $\{\mathbf{g} *[\mathbf{f}(n)-\mathbf{h}(n)]\}$, ao selecionar as primeiras $L_{G}+L_{F}+1$ amostras de $\mathbf{c}_{\mathbf{e}}(n)$ [3].

O caminho de avanço $G(q, n)$ pode ser estimado com certa precisão a partir de seus sinais de entrada e saída por um método de identificação de sistemas como um filtro adaptativo. Assim, conhecendo $\mathbf{g}(n)$, o método calcula $[\mathbf{f}(n)-\mathbf{h}(n)]^{\wedge}$, uma estimativa de $[\mathbf{f}(n)-\mathbf{h}(n)]$, da seguinte maneira [3]

$$
[\mathbf{f}(n)-\mathbf{h}(n)]^{\widehat{ }}=\{\mathbf{g}(n) *[\mathbf{f}(n)-\mathbf{h}(n)]\}^{\wedge} * \mathbf{g}^{-1}(n) .
$$

Após isso, o método calcula $\hat{\mathbf{f}}(n)$, uma estimativa de $\mathbf{f}(n)$, a partir de (12) como se segue [3]

$$
\hat{\mathbf{f}}(n)=[\mathbf{f}(n)-\mathbf{h}(n)]^{\widehat{ }}+\mathbf{h}(n-1)
$$

E, por fim, o método atualiza o filtro adaptativo como [3]

$$
\mathbf{h}(n)=\lambda \mathbf{h}(n-1)+(1-\lambda) \hat{\mathbf{f}}(n),
$$

onde $0 \leq \lambda<1$ é um fator de ponderação exponencial, denominado fator de esquecimento por fazer as estimativas mais antigas terem um efeito cada vez mais insignificante, que controla o compromisso entre robustez a perturbações de curta duração e capacidade de rastreamento do filtro adaptativo.

Portanto, a cada execução, o método AFC-CE obtém uma estimativa de $\mathbf{f}(n)$ para atualizar $\mathbf{h}(n)$. Se $\mathbf{f}(n)$ variar lentamente ao longo do tempo, esse esforço computacional pode não ser vantajoso, em relação ao desempenho, se for realizado a cada amostra de $e(n)$. Por isso, na prática, o método é executado apenas a cada $N_{f r}$ amostras, onde $N_{f r}$ é o parâmetro que controla o compromisso entre desempenho (latência e capacidade de rastreamento) e complexidade computational [3]. E, para evitar estimativas iniciais imprecisas, o método é executado apenas para $n \geq n_{0}$.

\section{Melhoria Proposta no Método AFC-CE}

Resultados simulados para $L_{f r}=8000, N_{f r}=1000$, $n_{0}=2000$ e $f_{s}=16 \mathrm{kHz}$, como proposto em [3], e a configuração de ambiente descrita na Seção IV demonstraram que o método AFC-CE apresenta desempenho satisfatório, no que diz respeito à velocidade de convergência e limite de desalinhamento, para $0,95 \leq \lambda \leq 0,99$. Resultados do método para valores de $\lambda$ nesse intervalo podem ser vistos na Figura 3. 
Esses resultados também demonstraram que quanto menor for $\lambda$, maior serão a velocidade de convergência e o limite de desalinhamento. Essa característica do método AFC-CE é, de certa forma, indesejada porque é de interesse combinar alta velocidade de convergência e baixo limite de desalinhamento.

Com o intuito de atingir essa combinação e assim melhorar o desempenho do método AFC-CE, esse trabalho propõe um algoritmo para transformar o fator de esquecimento $\lambda$ em $\lambda(n)$, isto é, variante no tempo. Esse algoritmo será baseado na

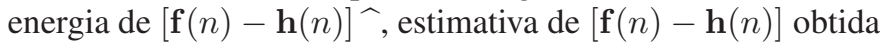
pelo método de acordo com (12), a qual será constantemente calculada para monitorar o desempenho do método.

Se $n=n_{0}$ ou essa energia for superior a $\gamma$, onde $n_{0}$ é o instante da primeira execução do método e $\gamma$ é um valor prédefinido, assume-se que $\mathbf{h}(n)$ não condiz com o caminho de realimentação a ser estimado e faz-se $\lambda=0,95$ com o intuito de aumentar a capacidade de rastreamento do filtro adaptativo e melhorar a sua estimativa de $\mathbf{f}(n)$. Espera-se que este caso ocorra quando houver uma abrupta variação em $\mathbf{f}(n)$.

Mas se $n>n_{0}$ e essa energia for inferior a $\gamma$, o valor de $\lambda$ é aumentado na sequência $0,95 \rightarrow 0,96 \rightarrow 0,97 \rightarrow 0,98 \rightarrow$ 0,985 com o intuito de melhorar o desalinhamento do método. Esse aumento é realizado de forma que o método opere com cada valor de $\lambda$ algumas vezes seguidas, onde a quantidade de execuções com cada valor depende do próprio valor de $\lambda$. O valor de $\lambda=0,99$ não é utilizado porque, apesar de melhorar o desempenho para $\Delta K=0$, pode deteriorar o desempenho do método para $\Delta K>0$ como será demonstrado na seção V-C.

O pseudo-código do algoritmo proposto para $\lambda(n)$ é apresentado no Algoritmo 1. O valor de $\gamma$ depende do $\mathbf{f}(n)$ a ser estimado enquanto os valores de $c_{1}, c_{2}, c_{3}$ e $c_{4}$ dependem de $N_{f r}$ e $f_{s}$. Para $N_{f r}=1000$ e $f_{s}=16 \mathrm{kHz}$, como proposto em [3], e o caminho de realimentação acústica descrito na seção IV-A.1, este trabalho propõe $\gamma=1,125, c_{1}=2, c_{2}=4$, $c_{3}=6$ e $c_{4}=60$.

\section{ConfiguraçÃo das SimulaçÕeS}

\section{A. Ambiente de Simulação}

1) Caminho de Realimentação: Duas respostas ao impulso da mesma sala, disponíveis em [13], foram utilizadas como respostas ao impulso do caminho de realimentação acústica. Elas tiveram suas frequências de amostragem reduzidas para $f_{s}=16 \mathrm{kHz}$ e foram truncadas de forma que $L_{F}=1000$. As resultantes $\mathbf{f}_{\mathbf{1}}$ e $\mathbf{f}_{\mathbf{2}}$ são exibidas na Figura 2 .

2) Caminho de Avanço: Como em [2], [3], o caminho de avanço foi definido como um atraso de $25 \mathrm{~ms}$ e um ganho, isto é,

$$
G(q, n)=g_{400}(n) q^{-400} .
$$

Então, de acordo com (7), $K(n)=g_{400}(n)$ e $J(q, n)=q^{-400}$.

O ganho de banda larga $K(n)$ foi inicializado com um valor $K_{1}$ tal que o sistema tivesse uma margem de ganho estável inicial igual a $5 \mathrm{~dB}$ para o método AFC-CE operar em uma condição de estabilidade e, assim, $H(q, n)$ convergir.

Em uma primeira configuração, $K(n)=K_{1}$ durante toda a simulação. Em uma segunda configuração, $K(n)=K_{1}$ até $5 \mathrm{~s}$ e em seguida $20 \log _{10} K(n)$ foi aumentado, a uma taxa de $1 \mathrm{~dB} / \mathrm{s}$, até $20 \log _{10} K_{2}$ de forma que $20 \log _{10} K_{2}=$ $20 \log _{10} K_{1}+\Delta K$. Finalmente, $K(n)=K_{2}$ durante $10 \mathrm{~s}$ resultando em um tempo total igual a $T=(15+\Delta K) \mathrm{s}$.
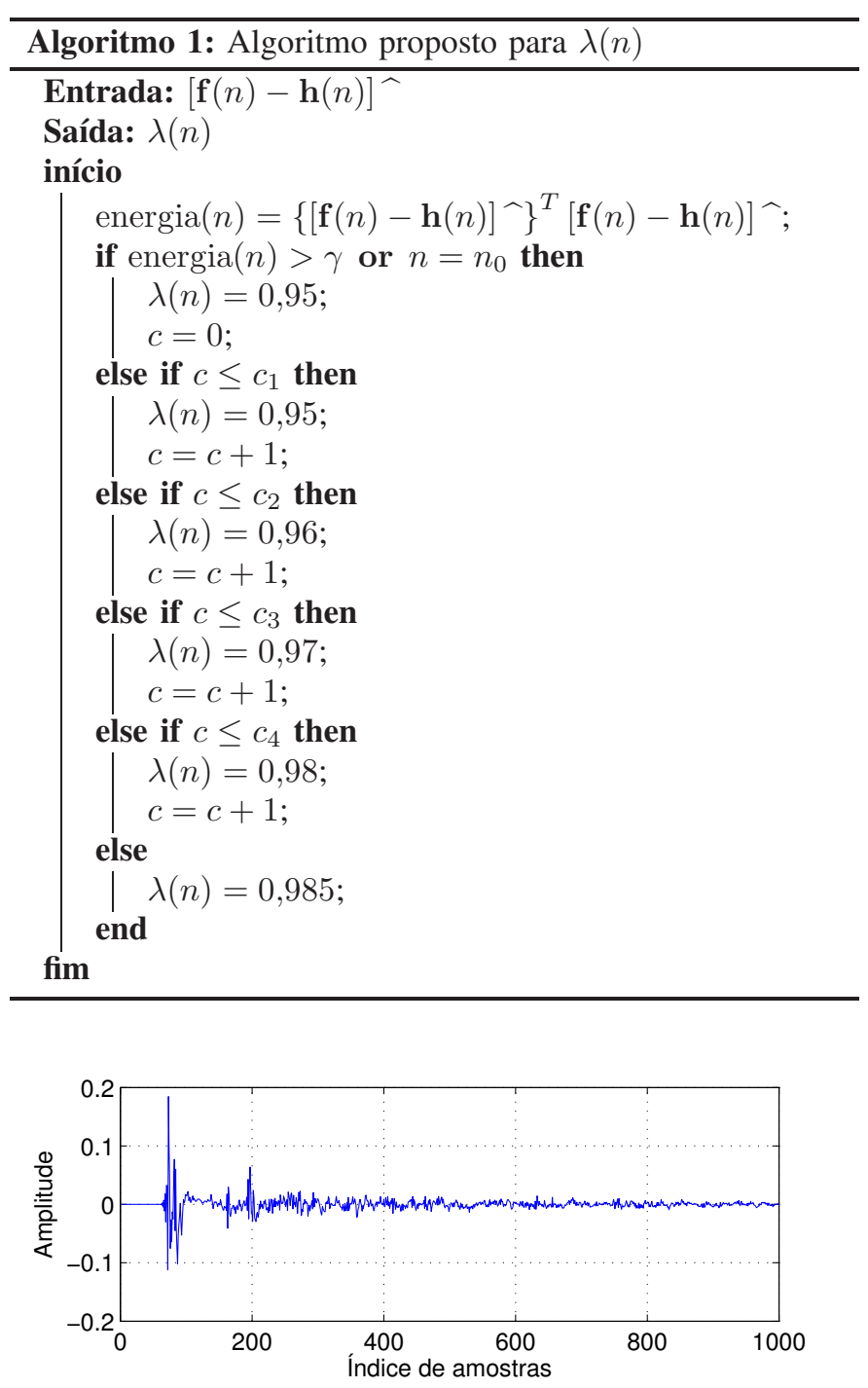

(a)

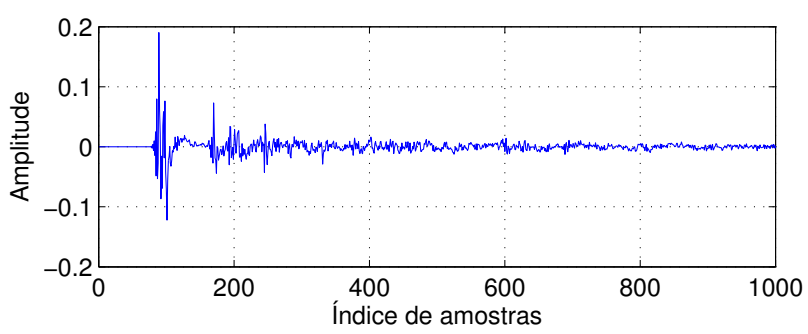

(b)

Fig. 2: Respostas ao impulso do caminho de realimentação: (a) $\mathrm{f}_{1}$; (b) $\mathrm{f}_{2}$.

\section{B. Métricas de Avaliação}

1) Desalinhamento: O desempenho da melhoria proposta no método AFC-CE foi avaliado por meio do desalinhamento normalizado (MIS), o qual é definido como [3]

$\operatorname{MIS}(n)=\frac{\|\mathbf{f}(n)-\mathbf{h}(n)\|}{\|\mathbf{f}(n)\|}=\frac{\left\|F\left(e^{j \omega}, n\right)-H\left(e^{j \omega}, n\right)\right\|}{\left\|F\left(e^{j \omega}, n\right)\right\|}$. 
2) Máximo Ganho Estável: O principal objetivo dos métodos AFC é aumentar o MSG do sistema de sonorização, o qual tem um limite superior devido à realimentação acústica. Portanto, o desempenho da melhoria proposta no método AFCCE também foi avaliado por meio do MSG do sistema com a inclusão do filtro adaptativo, o qual é definido como [2], [3]

$$
\begin{aligned}
& \operatorname{MSG}(n)(\mathrm{dB})=-20 \log _{10} \\
& \max _{\omega \in P_{H}(n)}\left|J\left(e^{j \omega}, n\right)\left[F\left(e^{j \omega}, n\right)-H\left(e^{j \omega}, n\right)\right]\right|,
\end{aligned}
$$

onde $P_{H}(n)$ denota o seguinte conjunto de frequências

$$
\begin{aligned}
P_{H}(n)=\left\{\omega \mid \angle G\left(e^{j \omega}, n\right)\left[F\left(e^{j \omega}, n\right)\right.\right. & \left.-H\left(e^{j \omega}, n\right)\right] \\
& =2 k \pi, k \in \mathbb{Z}\} .
\end{aligned}
$$

Vale ressaltar que as métricas $\operatorname{MIS}(n)$ e $\operatorname{MSG}(n)$ são relacionadas porque ambas dependem de $\left|F\left(e^{j \omega}, n\right)-H\left(e^{j \omega}, n\right)\right|$. No que diz respeito a esse fator, a diferença é que $\operatorname{MIS}(n)$ considera todas as frequências enquanto $\operatorname{MSG}(n)$ somente uma das frequências contidas em $P_{H}(n)$.

\section{Sinais de Voz}

Os sinais da fonte $v(n)$ foram criados a partir de sinais, ditos básicos, de um banco de sinais de voz. Cada sinal básico contém uma sentença curta gravada em um intervalo de tempo de $4 \mathrm{~s}$ e teve sua frequência de amostragem reduzida para $f_{s}=16 \mathrm{kHz}$. Todas as sentenças foram gravadas por locutores nativos, os quais tiveram as seguintes nacionalidades e gêneros: 4 Americanos ( 2 masculinos e 2 femininos), 2 Ingleses ( 1 masculino e 1 feminino), 2 Franceses (1 masculino e 1 feminino), 2 Alemães ( 1 masculino e 1 feminino).

Mas, como os experimentos necessitam de sinais com longa duração, vários sinais básicos do mesmo locutor foram concatenados e seus trechos de silêncio foram removidos, resultando assim em 10 sinais de voz (1 sinal por locutor).

\section{Resultado das SimulaçÕes}

\section{A. Experimento 1}

No primeiro experimento, um teste de convergência do método AFC-CE com $\lambda=\{0,95,0,96,0,97,0,98,0,99\}$ e com o algoritmo proposto para $\lambda(n)$ foi realizado ao se fazer $L_{H}=L_{F}, \mathbf{f}(n)=\mathbf{f}_{\mathbf{1}}$ e utilizar a primeira configuração de $K(n)$ onde $\Delta K=0$.

Os resultados são exibidos na Figura 3. Pode-se observar que o método com o algoritmo proposto para $\lambda(n)$ apresentou, de forma geral, o melhor desempenho durante os primeiros segundos de simulação (até $9 s$ no MIS e $7 s$ no MSG), sendo superado após isso somente pelo caso com $\lambda=0,99$. Isso deve-se ao fato do algoritmo proposto para $\lambda(n)$ não utilizar $\lambda=0,99$ como discutido na seção III e cujo motivo será abordado na seção V-C.

\section{B. Experimento 2}

No segundo experimento, um teste de re-convergência do método AFC-CE com $\lambda=\{0,95,0,96,0,97,0,98,0,99\}$ e com o algoritmo proposto para $\lambda(n)$ foi realizado ao se fazer $L_{H}=$ $L_{F}, \mathbf{f}(n)=\mathbf{f}_{\mathbf{1}}$ para $n<32000, \mathbf{f}(n)=\mathbf{f}_{\mathbf{2}}$ para $n \geq 32000 \mathrm{e}$ utilizar a primeira configuração de $K(n)$ onde $\Delta K=0$.

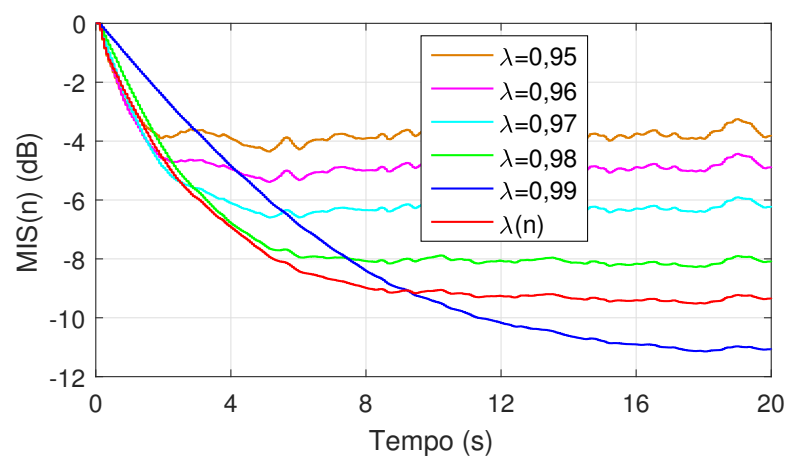

(a)

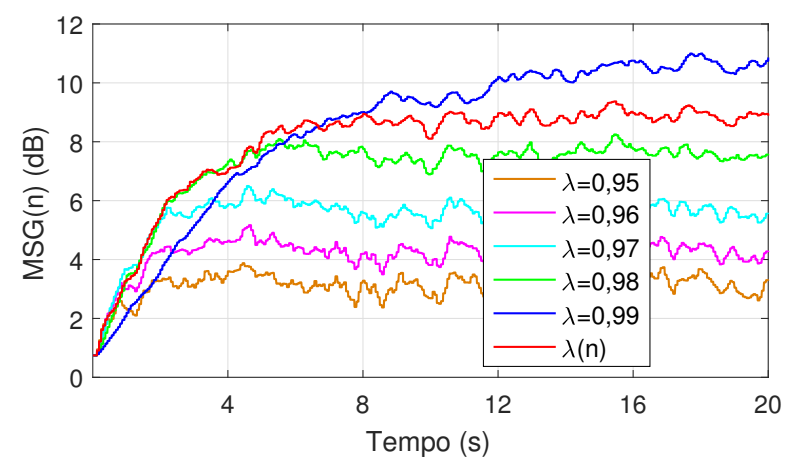

(b)

Fig. 3: Resultados do teste de convergência do método AFCCE para $\Delta K=0$ : (a) $\operatorname{MIS}(n)$; (b) $\operatorname{MSG}(n)$.

Os resultados são exibidos na Figura 4. Pode-se observar que o método com o algoritmo proposto para $\lambda(n)$ apresentou, de forma geral, o melhor desempenho durante os primeiros segundos de convergência (até $9 s$ no MIS e $7 s$ no MSG) e de re-convergência (entre 20 e $31 s$ no MIS e entre 20 e $28 s$ no MSG), sendo superado após esses momentos somente pelo caso com $\lambda=0,99$.

\section{Experimento 3}

No último experimento, um teste de convergência do método AFC-CE com $\lambda=\{0,95,0,96,0,97,0,98,0,99\}$ e com o algoritmo proposto para $\lambda(n)$ foi realizado ao se fazer $L_{H}=L_{F}, \mathbf{f}(n)=\mathbf{f}_{1}$ e utilizar a segunda configuração de $K(n)$ onde $\Delta K=30 \mathrm{~dB}$.

Os resultados são exibidos na Figura 5. Pode-se observar que o método com o algoritmo proposto para $\lambda(n)$ apresentou, de forma geral, o melhor desempenho até aproximadamente $20 \mathrm{~s}$ no MIS. Após esse instante de tempo, o método com $\lambda(n)$ foi superado durante alguns segundos pelo com $\lambda=0,99$. Porém, com $\lambda=0,99$, o sistema se torna instável entre $34 \mathrm{e}$ $40 s$ como pode ser observado na Figura 5b. Esse fato resultou na exclusão de $\lambda=0,99$ no algoritmo proposto para $\lambda(n)$.

No que diz respeito ao $\operatorname{MSG}(n)$, o método com o algoritmo proposto para $\lambda(n)$ apresentou, de forma geral, o melhor desempenho até aproximadamente $25 \mathrm{~s}$. O método com $\lambda(n)$ foi superado pelo com $\lambda=0,98$ e $\lambda=0,97$ após 25 e $30 \mathrm{~s}$, respectivamente. A discrepância de resultados no MIS e MSG se deve ao fato do primeiro considerar todas as frequências enquanto o segundo somente as contidas em $P_{H}(n)$, como discutido na seção IV-B. 


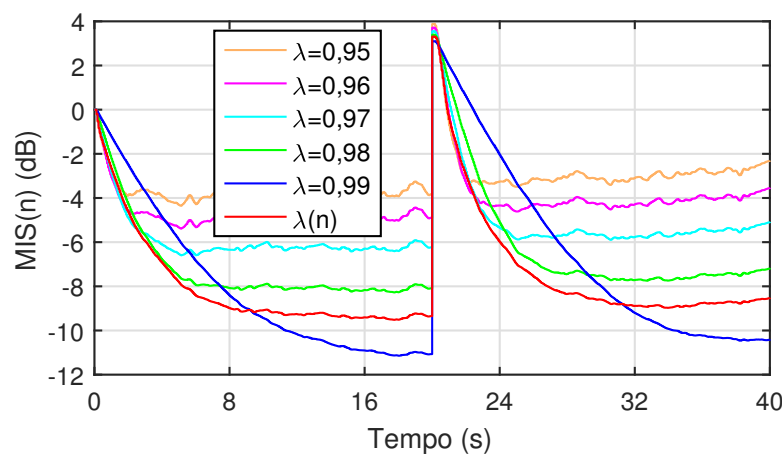

(a)

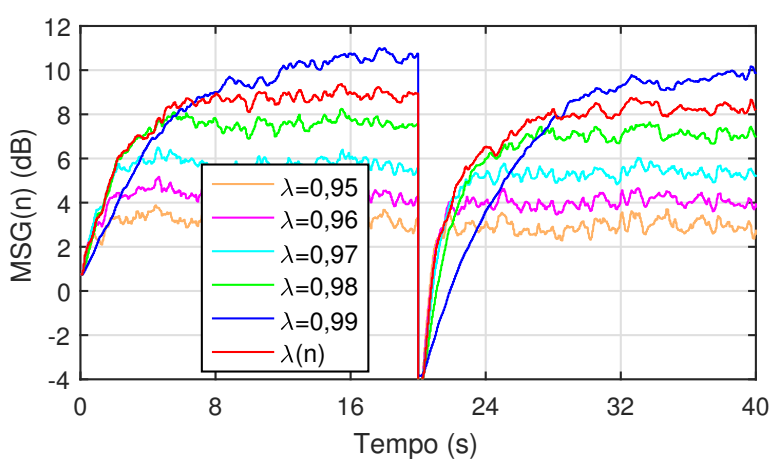

(b)

Fig. 4: Resultados do teste de re-convergência do método AFC-CE para $\Delta K=0$ : (a) $\operatorname{MIS}(n)$; (b) $\operatorname{MSG}(n)$.

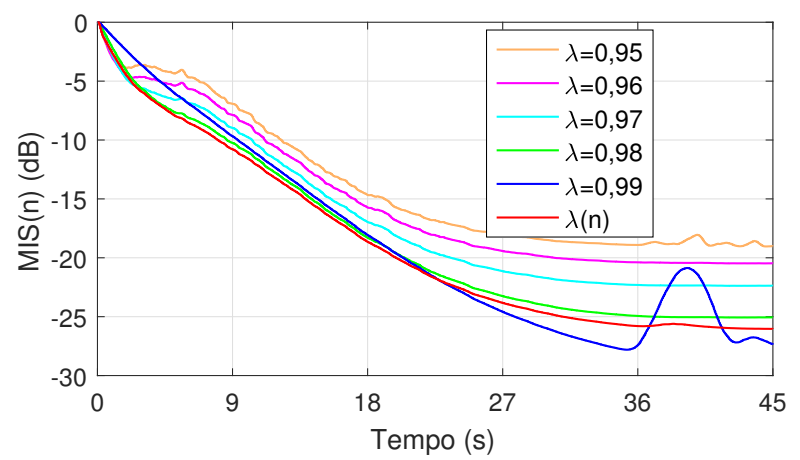

(a)

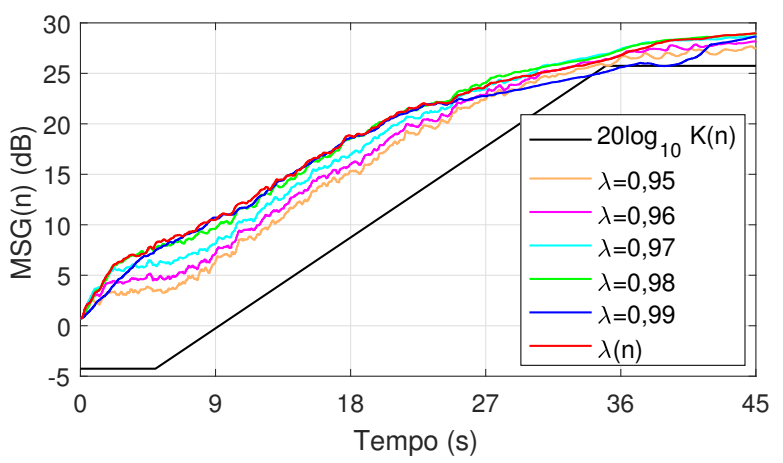

(b)

Fig. 5: Resultados do teste de convergência do método AFCCE para $\Delta K=30 \mathrm{~dB}$ : (a) $\operatorname{MIS}(n)$; (b) $\operatorname{MSG}(n)$.

\section{CONCLUSÕES}

Este trabalho propôs uma melhoria no do método de cancelamento de realimentação acústica baseado no cepstro do sinal de erro. Essa melhoria consistiu em transformar o fator de esquecimento, parâmetro que controla o compromisso entre robustez a perturbações de curta duração e capacidade de rastreamento do filtro adaptativo, em variante no tempo. Testes de convergência e re-convergência foram realizados em ambiente simulado utilizando sinais de voz. Resultados demonstraram que o algoritmo proposto faz o método apresentar um melhor compromisso entre velocidade de (re-)convergência e limite de desalinhamento, aumentando de maneira geral a margem de estabilidade do sistema de sonorização.

\section{AGRADECIMENTOS}

Os autores gostariam de agradecer à Fundação Araucária, à Secretaria de Estado da Ciência, Tecnologia e Ensino Superior (SETI-PR) e ao Governo do Estado do Paraná, pelo apoio financeiro recebido para viabilizar este trabalho.

\section{REFERÊNCIAS}

[1] L. Ljung, System Identification: Theory for the User, 2nd ed. Upper Saddle River, New Jersey: Prentice Hall, 1999.

[2] T. van Waterschoot and M. Moonen, "Fifty years of acoustic feedback control: state of the art and future challenges," Proceedings of the IEEE, vol. 99, no. 2, pp. 288-327, February 2011.

[3] B. C. Bispo and D. R. S. Freitas, "On the use of cepstral analysis in acoustic feedback cancellation," Digital Signal Processing, vol. 44, pp. 88-101, September 2015.

[4] B. C. Bispo and S. L. Netto, "Comparação de algoritmos adaptativos para cancelamento de eco acústico," in XXV Simpósio Brasileiro de Telecomunicações, Recife, Brasil, Setembro 2007.

[5] J. Hellgren and U. Forssell, "Bias of feedback cancellation algorithms in hearing aids based on direct closed loop identification," IEEE Transactions on Speech and Audio Processing, vol. 9, no. 7, pp. 906-913, November 2001.

[6] M. G. Siqueira and A. Alwan, "Steady-state analysis of continuous adaptation in acoustic feedback reduction systems for hearing-aids," IEEE Transactions on Speech and Audio Processing, vol. 8, no. 4, pp. 443-453, July 2000.

[7] C. F. Yamamura, W. M. da Silva Nogueira, and B. C. Bispo, "Análise de mínimos quadrados do cancelamento de realimentação acústica," in Proceedings of XXXV Simpósio Brasileiro de Telecomunicações, São Pedro, Brazil, Septembter 2017, pp. 742-746.

[8] B. C. Bispo, P. M. L. Rodrigues, and D. R. S. Freitas, "Acoustic feedback cancellation based on cepstral analysis," in Proceedings of 17th IEEE Conference on Signal Processing Algorithms, Architectures, Arrangements and Applications, Poznan, Poland, September 2013, pp. 205-209.

[9] D. R. S. Freitas and B. C. Bispo, "Acoustic feedback cancellation based on cepstral analysis," WIPO WO 2015/044915A1, 2015.

[10] B. C. Bispo and D. R. S. Freitas, "Evaluation of acoustic feedback cancellation methods with multiple feedback paths," in Proceedings of International Conference on Signal Processing and Multimedia Applications, Vienna, Austria, August 2014, pp. 127-133.

[11] _ _ "Performance evaluation of acoustic feedback cancellation methods in single-microphone and multiple-loudspeakers public address systems," in e-Business and Telecommunications - Communications in Computer and Information Science, M. S. Obaidat, A. Holzinger, and J. Filipe, Eds. Springer, 2015, vol. 554, pp. 473-495.

[12] B. C. Bispo, J. N. Moutinho, P. M. L. Rodrigues, and D. R. S. Freitas, "Assessing the cepstrum-based acoustic feedback cancellation method for short acoustic paths," in Proceedings of 12th IEEE International Conference on Industry Applications, Curitiba, Brazil, November 2016, pp. 1-6.

[13] M. Jeub, M. Schäfer, and P. Vary, "A binaural room impulse response database for the evaluation of dereverberation algorithms," in Proceedings of International Conference on Digital Signal Processing, Santorini, Greece, July 2009. 